\title{
Nucleolin antisense oligodeoxynucleotides induce apoptosis and may be used as a potential drug for nasopharyngeal carcinoma therapy
}

\author{
CHENG-DER WU ${ }^{1}$, HUNG-WEN CHOU ${ }^{1}$, YUAN-SUNG KUO ${ }^{1,3}$, RUEI-MIN LU ${ }^{1}$, \\ YU-CHYI HWANG ${ }^{1}$, HAN-CHUNG WU ${ }^{1,4}$ and CHIN-TARNG LIN ${ }^{1,2}$ \\ ${ }^{1}$ Institute of Pathology, College of Medicine, National Taiwan University; Departments of \\ ${ }^{2}$ Pathology, ${ }^{3}$ Surgery, National Taiwan University Hospital, Taipei 10002; ${ }^{4}$ Institute of Cellular \\ and Organismic Biology, Academia Sinica, Taipei 11529, Taiwan, R.O.C.
}

Received July 7, 2011; Accepted August 1, 2011

DOI: 10.3892/or.2011.1476

\begin{abstract}
Nucleolin (C23, NCL) mRNA was up-regulated in nasopharyngeal carcinoma (NPC) cells compared to that of normal nasomucosal (NNM) cells using a cDNA microarray approach. The level of nucleolin protein was also up-regulated in 13 NPC cell lines, 30 biopsy specimens and nine other cancer cell lines compared to five NNM cells or normal stromal cells, which were analyzed using immunoblotting or immunohistochemistry. We transfected nucleolin antisense oligodeoxynucleotides (phosphorothioate-modified oligodeoxynucleotides; S-ODNs) into NPC-TW01 cells to knockdown nucleolin expression to evaluate the function of nucleolin in cancer cells. Nucleolin knockdown induced NPC cells but not NNM cells to undergo apoptosis. Furthermore, treatment of NPC-TW01 xenograft tumors with nucleolin antisense oligodeoxynucleotides suppressed the growth of xenograft tumors without obvious side effects. Therefore, we suggest that nucleolin may be a potential cancer therapeutic target and that nucleolin antisense oligodeoxynucleotides may be used as a potential drug for therapy in NPC.
\end{abstract}

\section{Introduction}

Cancer is the one of the top 10 causes of death in developed countries. The trends for cancer case numbers, morbidity and

Correspondence to: Dr Chin-Tarng Lin, Department of Pathology, College of Medicine, National Taiwan University Hospital, No. 7, Chung-Shan S. Rd., Taipei 10002, Taiwan, R.O.C.

E-mail: ctl@ntu.edu.tw

Dr Han-Chung Wu, Institute of Cellular and Organismic Biology, Academia Sinica, No. 128, Academia Rd., Section 2, Nankang, Taipei 11529, Taiwan, R.O.C.

E-mail: hcw0928@gate.sinica.edu.tw

Key words: nasopharyngeal carcinoma, apoptosis, nucleolin, antisense oligodeoxynucleotides mortality are rising in developing countries. Cancer caused 7.6 million deaths $(\sim 13 \%$ of all deaths) in the world in 2008 (1). The fight against cancer is a worldwide issue. To obtain a better understanding of the characteristics of cancer, we have previously established ten nasopharyngeal carcinoma (NPC) cell lines from keratinizing squamous cell carcinoma and undifferentiated carcinoma, which are designated as NPC-TW01 to NPC-TW10. All of the carcinomas were derived from Chinese patients living in Taiwan $(2,3)$. To define the alteration of gene expression between NPC cells and normal cells, mRNA from NPC cells and normal cells was previously analyzed using cDNA microarray (4), which showed that nucleolin is markedly up-regulated in NPC cells.

Nucleolin, which is also known as C23 or NCL, is a phosphoprotein with many functions, such as acting as an RNA-binding protein, an autoantigen, a transcriptional repressor, a DNA and RNA helicase as well as a DNA-dependent ATPase $(5,6)$. The synthesis of nucleolin is positively correlated with increased rates of cell division. Therefore, nucleolin levels are high in tumors or other rapidly dividing cells and are present at low levels in normal or quiescent cells (7-10). Nucleolin may play an important role in tumorigenesis. Therefore, we used nucleolin antisense oligodeoxynucleotides to knockdown nucleolin expression to determine the function of nucleolin in NPC cells.

Antisense strategies such as ribozymes, oligodeoxynucleotides (ODNs), microRNA (miRNA) and small interfering RNA (siRNA) can be used for cancer therapy $(11,12)$. The oligodeoxynucleotide antisense method is a well-built technology in our laboratory (13) and is currently one of advanced treatments in drug clinical trials (14-17). In the present study, we investigated the nucleolin mRNA and protein expression in NPC cell lines, biopsy specimens and other cancer cell lines. Since nucleolin expression was up-regulated in the cell lines and biopsy specimens, we used nucleolin antisense oligodeoxynucleotides to knock down nucleolin. Nucleolin knockdown by nucleolin antisense oligodeoxynucleotides induced apoptosis in cancer cells but not in normal cells. Treatment with nucleolin antisense oligodeoxynucleotides suppressed xenograft tumor growth. Therefore, nucleolin and nucleolin antisense 
oligodeoxynucleotides may have potential applications in nasopharyngeal carcinoma therapy.

\section{Materials and methods}

Cell lines. The following cell lines were used for this study: NPC-TW01 to NPC-TW10, which are nasopharyngeal carcinoma cell lines that were established in our laboratory $(2,3)$; NPC-BM-1, which is a bone marrow metastatic nasopharyngeal carcinoma cell line that was established by Dr S.K. Liau of Chang-Gung University (18); NPC-HONE1 (19) and NPC-CNE-1 (20), which are nasopharyngeal carcinoma cell lines that were originally established in China; and NNM-9, $-11,-12,-13$ and -14 , which are five primary cultures of normal nasomucosal epithelia that were established in our laboratory from nasal polyps provided by Dr C.M. Liu in the Department of Otolaryngology at the National Taiwan University Hospital. The culture conditions were based on our previously published method (3). The following cell lines were also used: SKOV3, which is a human ovarian cancer cell line (ATCC HTB-77); SW620, which is a human colon cancer cell line (ATCC CCL-227); SAS, which is a human tongue carcinoma cell line (JCRB0260); Ca 9-22, which is a human gingival carcinoma cell line (JCRB0625); Cal-27, which is a human tongue carcinoma cell line (ATCC CRL-2095); THP-1, which is a human acute monocytic leukemia cell line (ATCC TIB-202); Ca Ski, which is a human cervical carcinoma cell line (ATCC CRM-CRL-1550); CL1-5, which is a human pulmonary adenocarcinoma cell line (21); and PC-3, which is a human prostate carcinoma cell line (ATCC CRL-1435). The use of nasal polyps and human cancer cell lines was approved by the IRB at the National Taiwan University Hospital, Taiwan.

Immunohistochemical and histopathological staining. The paraffin sections of NPC biopsy specimens were immunostained using the mouse monoclonal antibody against human nucleolin, which was kindly provided by Dr L.S. Yeh of the Institute of Immunology at the College of Life Science at the National Yang-Ming University, Taiwan (22), according to routine immunoperoxidase procedures. The paraffin sections from nucleolin antisense and sense oligodeoxynucleotidetreated NPC xenograft tumors were subjected to hematoxylin and eosin (H\&E) staining.

Transfection of nucleolin antisense and sense oligodeoxynucleotides. The nucleolin antisense oligodeoxynucleotides (5'-atgatggcggcggagtgtga-3') and sense oligodeoxynucleotides (5'-tcacactccgccgccatcat-3') were phosphorothioate-modified oligodeoxynucleotides (S-ODNs). They were synthesized by Sigma-Genosys (Sigma-Aldrich, MO, USA). A total of $1.5 \times 10^{5}$ cells were seeded in each well of a 6 -well culture plate in $1 \mathrm{ml}$ of normal culture medium for oligodeoxynucleotide transfection. The oligodeoxynucleotide transfection mixture was prepared by diluting $1 \mu \mathrm{l}$ of $50 \mu \mathrm{M}$ oligodeoxynucleotides in $100 \mu \mathrm{l}$ of Opti-MEM I reduced serum medium (Gibco, Gaithersburg, MD, USA). A total of $6 \mu \mathrm{l}$ of HiPerFect transfection reagent (Qiagen, Valencia, CA, USA) was added to the diluted oligodeoxynucleotides and mixed by vortexing. After incubating for $10 \mathrm{~min}$ at room temperature, normal culture medium was added to the mixture to bring the final volume to $1 \mathrm{ml}$. The oligodeoxynucleotide transfection mixture was added to the well containing cells and mixed gently. The culture medium in all of the experimental groups was changed after $24 \mathrm{~h}$ to normal culture medium and re-transfected.

Quantitative RT-PCR. For mRNA quantification, total RNA was extracted using TRIzol (Invitrogen, Gaithersburg, MD, USA) and reverse transcribed using Superscript III (Invitrogen). The quantitative real-time polymerase chain reaction (qRTPCR) experiments were performed using the ABI Power SYBR Green PCR Master mix with the ABI 7500 Real-Time PCR System (Applied Biosystems, Austin, TX, USA). All of the experimental protocols were performed according to the manufacturer's instructions. For qRT-PCR, we used the following primers: nucleolin-F 5'-gcacttggagtggtgaatcaaa-3', nucleolin-R 5'-aaatgcataccctttagatttgcc-3'; GAPDH-F 5'-gcaccgtcaaggc tgagaa-3', GAPDH-R 5'-agggatctcgctcctggaa-3'; $\beta$-actin-F 5 '-ctcaggaggagcaatgatcttgat-3', and $\beta$-actin-R 5'-acgtggacatcc gcaaagac-3'.

Apoptosis assay. The morphological features of cell apoptosis were observed using Astec CCM-Multi microscopy (Astec, Fukuoka, Japan). Phosphatidylserine was stained using an Annexin V-FITC assay kit (Serotec, Raleigh, USA). DNA fragments were stained using a DeadEnd Fluorometric TUNEL system (Promega, Madison, WI, USA). Annexin V and TUNEL staining were imaged using Zeiss Axiovert $200 \mathrm{M}$ fluorescence microscopes and analyzed using MetaMorph 7.0 software. All of the experimental processes were performed according to the manufacturer's instructions.

Animal experiments. SCID mice (females, 4-6 weeks old) were purchased from the Laboratory Animal Center at the National Taiwan University Hospital. Cultured NPC-TW01 cells were subcutaneously injected ( $1 \times 10^{7}$ cells) into the right flank. When the xenograft tumor reached a size of $0.5-0.7 \mathrm{~cm}^{3}$, the mice were randomly divided into three groups as follows: antisense, sense and PBS. Each group contained four mice. Each of the first two groups was intravenously injected every 2 days for 4 weeks through the tail vein with $10 \mathrm{mg} / \mathrm{kg}$ of antisense or sense oligodeoxynucleotides, respectively. The control group was injected with PBS solution. Animals were inspected daily for tumor development. Growing tumors were measured using a caliper, and the tumor volume was calculated using the following formula: length $\mathrm{x}$ width ${ }^{2} \mathrm{x} 0.52$, which approximates the volume of an elliptical solid mass. The animals were sacrificed at day 38. The xenograft tumors were examined using qRT-PCR analysis, immunoblotting or histopathological observation. The use of animals was approved by the Institutional Animal Care and Use Committee at the College of Medicine at the National Taiwan University.

\section{Results}

Nucleolin is up-regulated in NPC cell lines, biopsy specimens and other cancer cell lines. Previously, we analyzed the mRNA expression profiles between nasopharyngeal carcinoma (NPC) cells and normal nasomucosal (NNM) cells using a cDNA microarray approach. Since nucleolin mRNA was up-regulated in NPC cells, we tested whether the level of nucleolin protein 
A

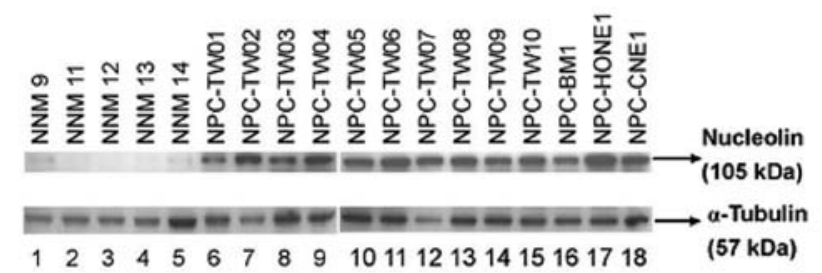

B

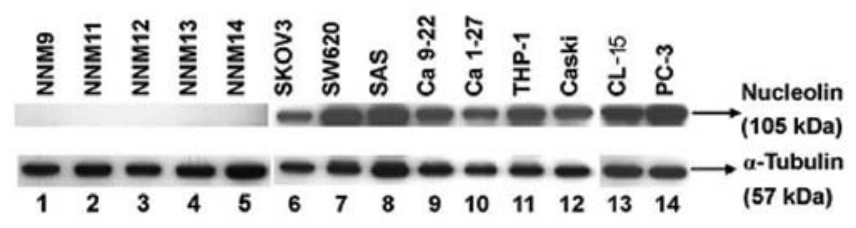

Figure 1. Immunoblot analyses of nucleolin protein expression in nasopharyngeal carcinoma (NPC) cell lines, normal nasomucosal (NNM) cells and other cancer cell lines. (A) All of the 13 NPC cell lines reveal strong bands indicating nucleolin protein expression, whereas five NNM cell lines have undetectable or weak bands. (B) Nucleolin protein levels in nine cancer cell lines are stronger compared to those of five NNM cell lines. The 57-kDa band of $\alpha$-tubulin was used as an internal control.

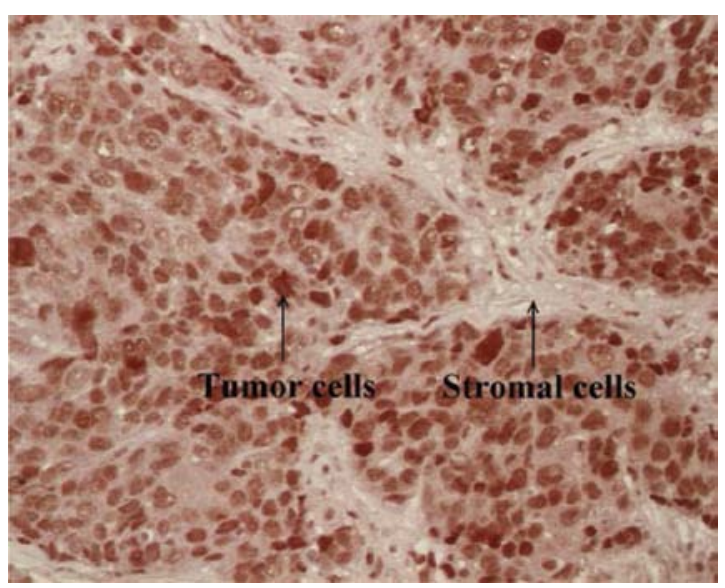

Figure 2. Immunohistochemistry showing nucleolin protein staining in human nasopharyngeal carcinoma (NPC) biopsy specimen. Most tumor cells in the NPC biopsy specimen display strong nucleolin reaction products, whereas the normal stromal cells show weak staining or unstained areas.

was also up-regulated in NPC cell lines and in other cancer cell lines using immunoblotting. The nucleolin protein levels in 13 NPC cell lines (Fig. 1A) and nine other cancer cell lines (Fig. 1B) were up-regulated compared to those of five NNM cells. To verify the up-regulation of nucleolin protein expression in human cancer tissue, immunohistochemistry against nucleolin was performed in 30 NPC biopsy specimens. Nucleolin protein was strongly expressed in tumor cells but weakly or not expressed in normal stromal cells (Fig. 2).

Nucleolin antisense oligodeoxynucleotides knock down the nucleolin expression and decrease viability in NPC-TWO1 cells, but not NNM cells. Since nucleolin expression was up-regulated in NPC cancer cells, we knocked down nucleolin using nucle-
A

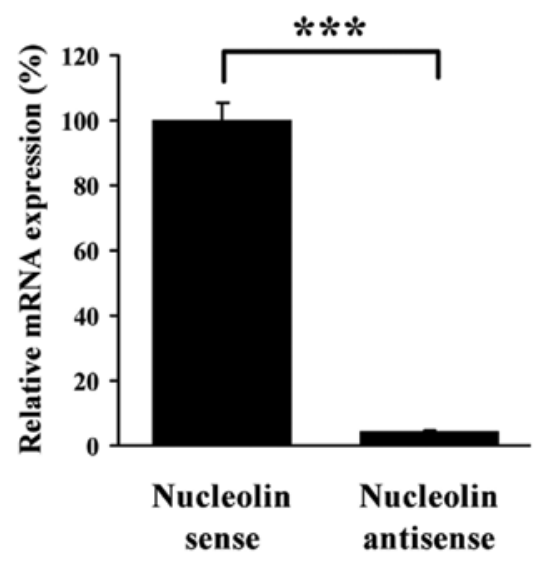

B

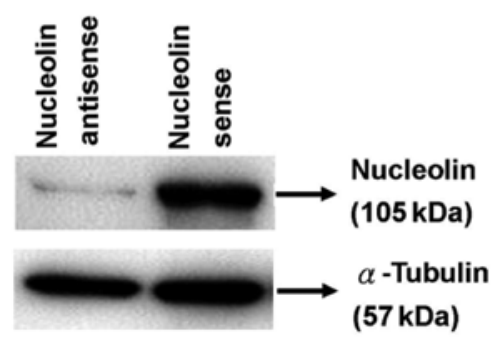

Figure 3. Transfection of nucleolin antisense oligodeoxynucleotides downregulates nucleolin mRNA and protein. NPC-TW01 cells were transfected with nucleolin antisense or sense (negative control) oligodeoxynucleotides. After transfection for $24 \mathrm{~h}$, total RNA was isolated from these cells, quantitated and compared using qRT-PCR, normalized to GAPDH mRNA (A) After $48 \mathrm{~h}$, the total proteins were analyzed by immunoblotting (B). The results show that nucleolin antisense oligodeoxynucleotides dramatically inhibited nucleolin mRNA and protein expression. ${ }^{* * *}$ Comparison between two groups as indicated, $\mathrm{p}<0.001$. Error bars correspond to the mean \pm SD.

olin antisense oligodeoxynucleotides to observe the influence of down-regulated nucleolin in cancer cells. After NPC-TW01 cells were transfected with nucleolin antisense oligodeoxynucleotides, we analyzed nucleolin mRNA using qRT-PCR (Fig. 3A) and nucleolin protein using immunoblotting (Fig. 3B). The results show that nucleolin antisense oligodeoxynucleotides dramatically knocked down nucleolin mRNA and protein expression. After the transfection of nucleolin antisense oligodeoxynucleotides, we demonstrated that nucleolin antisense oligodeoxynucleotides, but not nucleolin sense oligodeoxynucleotide, affected the viability of NPC-TW01 cells (Fig. 4A). We tested other cell lines such as NPC-TW02, NPC-TW06, SKOV3, SW620, SAS and PC-3 cells, which confirmed that the transfection of nucleolin antisense oligodeoxynucleotides decreased cell viability (data not shown). Furthermore, nucleolin antisense oligodeoxynucleotides did not affect the viability of NNM cells (Fig. 4B).

Transfection of nucleolin antisense oligodeoxynucleotides induces NPC-TWO1 cells to undergo apoptosis. To investigate the mechanism of nucleolin antisense oligodeoxynucleotideinduced decreases in cell viability, NPC-TW01 cells were transfected with nucleolin antisense oligodeoxynucleotides and observed using microscopy. We found that apoptotic bodies were apparent in nucleolin antisense oligodeoxynucleotide- 
A

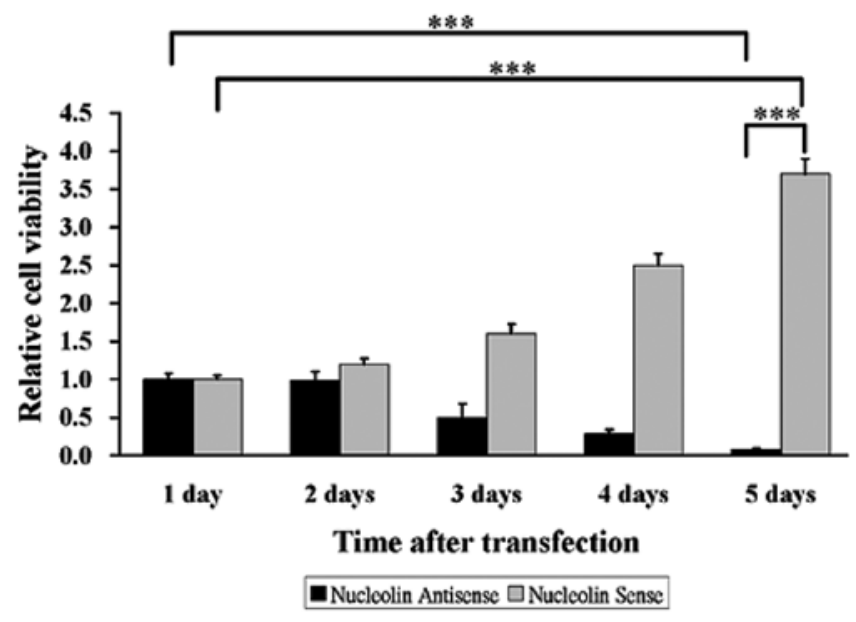

B

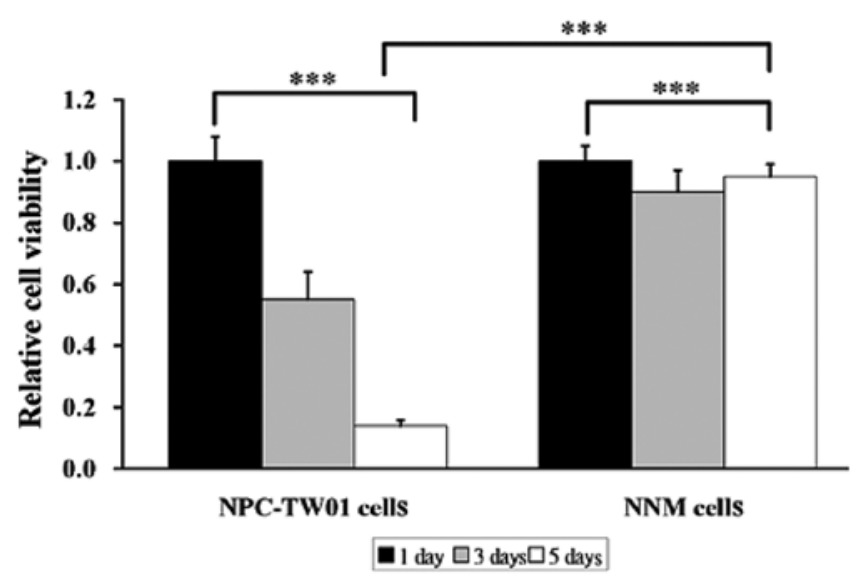

Figure 4. Nucleolin antisense oligodeoxynucleotides decrease cell viability in NPC-TW01 (nasopharyngeal carcinoma) cells but not NNM (normal nasomucosal) cells. (A) NPC-TW01 cells were transfected with nucleolin antisense or sense oligodeoxynucleotides. Cell viability was evaluated using the MTT assay at different time points. The comparison of cell viability at 1 and 5 days after transfection indicates that cell viability is reduced in nucleolin antisense oligodeoxynucleotide-transfected cells but not nucleolin sense oligodeoxynucleotide-transfected cells. The comparison of cell viability at 5 days after transfection demonstrates that nucleolin antisense oligodeoxynucleotides but not nucleolin sense oligodeoxynucleotides affect cell viability. ${ }^{* * *}$ Comparison between two groups as indicated, $\mathrm{p}<0.001$. Error bars correspond to the mean \pm SD. (B) NPC-TW01 cells or NNM cells were transfected with nucleolin antisense oligodeoxynucleotides. Cell viability was evaluated using the MTT assay at different time points. Nucleolin antisense oligodeoxynucleotides affect cell viability in NPC-TW01 cells bu not NNM cells. ${ }^{* * *}$ Comparison between two groups as indicated, $\mathrm{p}<0.001$. Error bars correspond to the mean $\pm \mathrm{SD}$

transfected cells (Fig. 5A). Furthermore, we confirmed the apoptosis by Annexin V and TUNEL staining. The nucleolin antisense oligodeoxynucleotide-transfected cells displayed Annexin V- and TUNEL-positive staining, whereas the nucleolin sense oligodeoxynucleotide-transfected cells and the untransfected cells showed Annexin V- and TUNELnegative staining (Fig. 5B and C). These results indicate that transfection of nucleolin antisense oligodeoxynucleotides may induce apoptosis in NPC-TW01 cells.
Nucleolin antisense oligodeoxynucleotides suppress NPC-TW01 xenograft tumor growth. To assess the effect of nucleolin antisense oligodeoxynucleotides on apoptosis in vivo and its potential application for cancer therapy, we implanted NPC-TW01 cells into SCID mice. The NPC-TW01 xenograft tumors were treated with nucleolin antisense oligodeoxynucleotides, nucleolin sense oligodeoxynucleotides and PBS. The results indicate that treatment with nucleolin antisense oligodeoxynucleotides suppresses NPC-TW01 xenograft tumor growth (Fig. 6A and B). The mice treated with nucleolin antisense oligodeoxynucleotides had no obvious side effects such as tiredness, nausea, loss of appetite, constipation, diarrhea or hair loss (Fig. 6C). The xenograft tumor sections were obtained from nucleolin antisense oligodeoxynucleotide-treated mice and observed after H\&E staining, which indicated marked necrosis and apoptotic tumor cells in the tumor (Fig. 6D). However, the nucleolin sense oligodeoxynucleotide-treated tumor sections showed many mitotic cells and few apoptotic cells in the tumor (Fig. 6E). Total RNA and proteins were extracted from the xenograft tumors that were treated with nucleolin antisense and sense oligodeoxynucleotides. We subsequently analyzed the nucleolin expression using qRT-PCR and immunoblotting. The results show that nucleolin mRNA and protein expression levels of nucleolin antisense oligodeoxynucleotide-treated xenograft tumors were down-regulated compared to those of nucleolin sense oligodeoxynucleotide-treated xenograft tumors (data not shown).

\section{Discussion}

In the analysis of microarray data from the NPC cells and normal cells (4), we found that nucleolin is a highly expressed gene in NPC cells. In addition, nucleolin expression levels are increased in many tumor cells and rapidly dividing cells (8-10). Therefore, we investigated whether nucleolin was also highly expressed in human NPC biopsy specimens. The results from our immunoblotting analysis indicate that nucleolin expression in different NPC cell lines, other cancer cell lines and biopsy specimens was up-regulated. However, normal and stromal cells revealed very weak or no expression of nucleolin. These findings suggest that nucleolin expression may be used as a pathologic diagnostic biomarker.

In the current study, we compared nucleolin antisense oligodeoxynucleotides with commercial nucleolin siRNA (Qiagen, cat. SI00300923). After transfection for $24 \mathrm{~h}$, we determined the down-regulation of nucleolin mRNA using qRT-PCR. The nucleolin antisense oligodeoxynucleotides and nucleolin siRNA had similar effects, knocking down nucleolin mRNA expression. Transfection of nucleolin sense oligodeoxynucleotides and negative control siRNA (Qiagen, cat. 1027281) did not affect nucleolin mRNA expression. Transfection of nucleolin antisense oligodeoxynucleotides down-regulated nucleolin mRNA, had no effect on $\beta$-actin and GAPDH mRNA and up-regulated TNF- $\alpha$ mRNA (data not shown). Therefore, our transfection conditions did not introduce off-target effects. Transfection of nucleolin antisense oligodeoxynucleotides up-regulated the mRNA of some pro-apoptotic genes such as TNF- $\alpha$. We intend to verify the pathway network between nucleolin and pro-apoptotic genes in the near future. 
A

Antisense

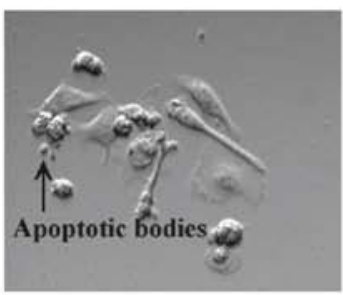

B
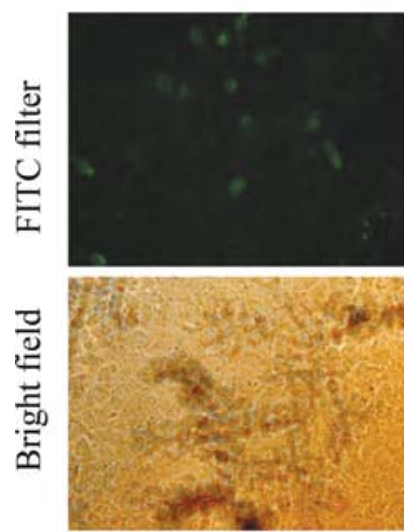

C Antisense
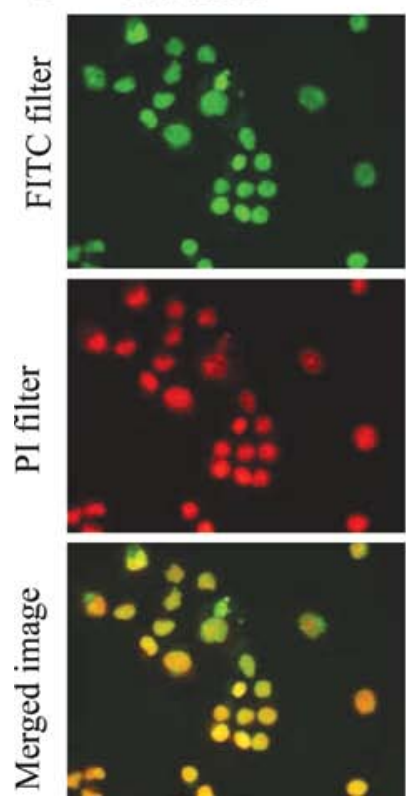

Sense

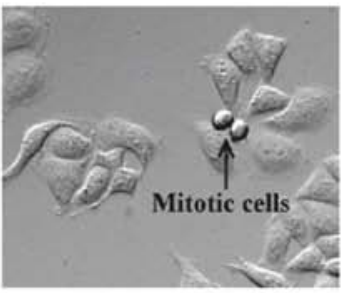

Sense
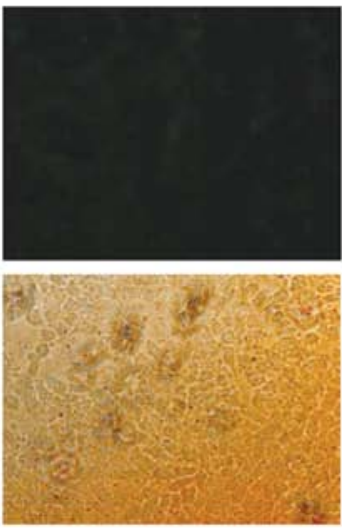

Sense
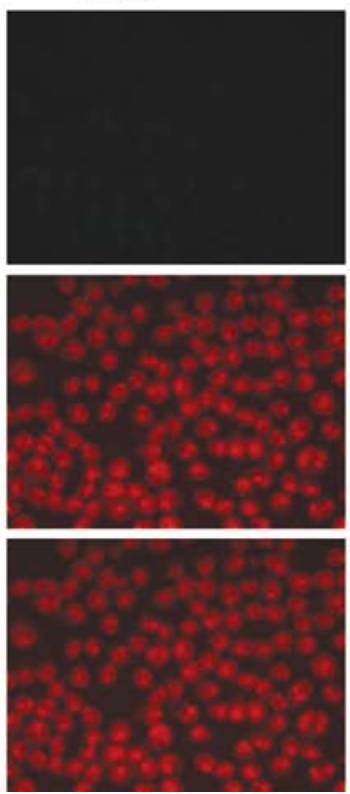

untransfected

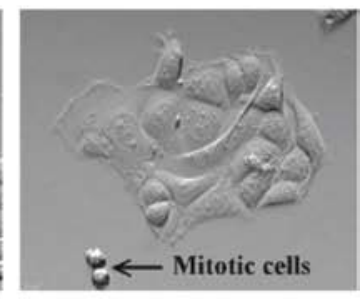

untransfected
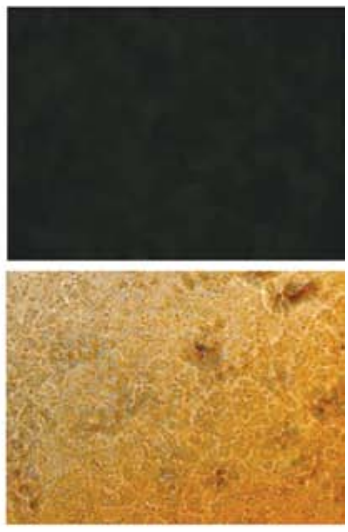

untransfected
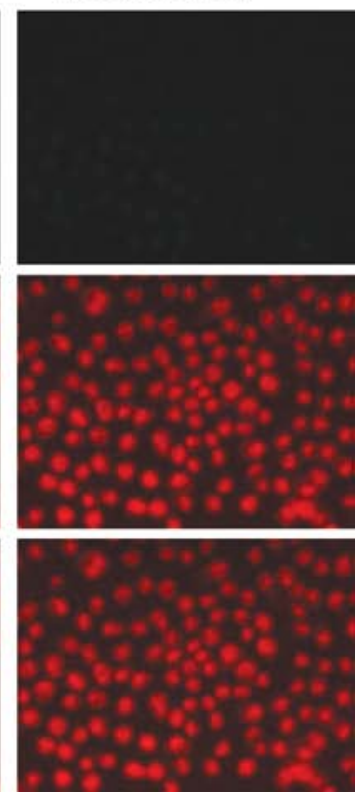

Figure 5. Transfection of nucleolin antisense oligodeoxynucleotides induces apoptosis in NPC-TW01 cells. From left to right: transfection with nucleolin antisense oligodeoxynucleotides, transfection with nucleolin sense oligodeoxynucleotides (negative control), and untransfected (blank control) NPC-TW01 cells. (A) The morphology was observed using microscopy after a 72-h transfection. Apoptotic bodies are apparent in nucleolin antisense oligodeoxynucleotidetransfected cells. (B) After transfection for $24 \mathrm{~h}$, the cells were subjected to Annexin V staining. (C) After transfection for $48 \mathrm{~h}$, the cells were subjected to TUNEL staining. The nucleolin antisense oligodeoxynucleotide-transfected cells display Annexin V- and TUNEL-positive staining, whereas the nucleolin sense oligodeoxynucleotide-transfected cells and untransfected cells show Annexin V- and TUNEL-negative staining.

In this study, transfection of nucleolin antisense oligodeoxynucleotides induced NPC-TW01 cells to undergo apoptosis. There are some reports that explain the molecular mechanism as follows: nucleolin stabilizes bcl-2 mRNA by binding bcl-2 mRNA 3'UTR AU-rich elements (23-25), nucleolin binds to p53 5'UTR and suppresses p53 translation and nucleolin down-regulation promotes $\mathrm{p} 53$ expression and induces apoptosis (26). In the absence of nucleolin, pre-ribosomal RNA and nucleolar structures accumulate, cell growth is arrested at the G2 phase, and the incidence of apoptosis increases (27).

Preliminary observations indicate that the mice treated with nucleolin antisense oligodeoxynucleotides displayed no obvious side effects. It is possible that some of the potential side effects were difficult to observe based on the external signs 
A

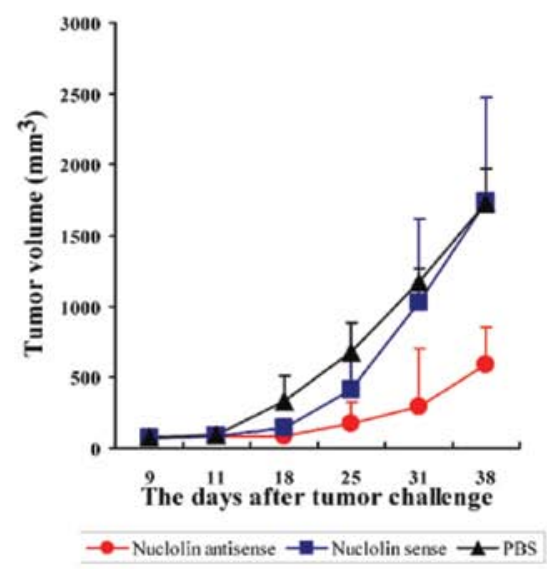

$\mathrm{C}$

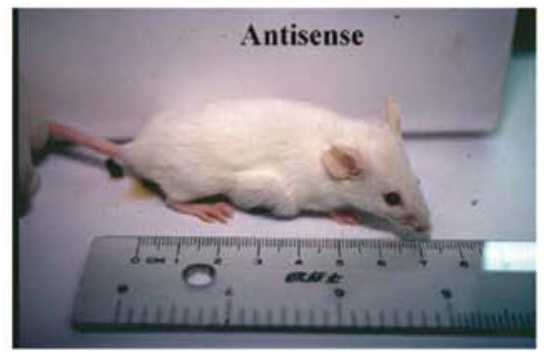

$\mathrm{D}$

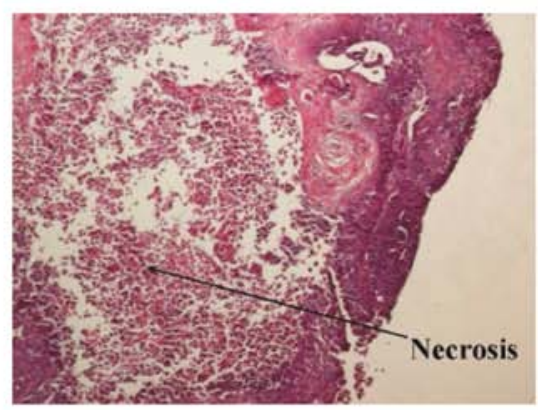

$\mathrm{E}$

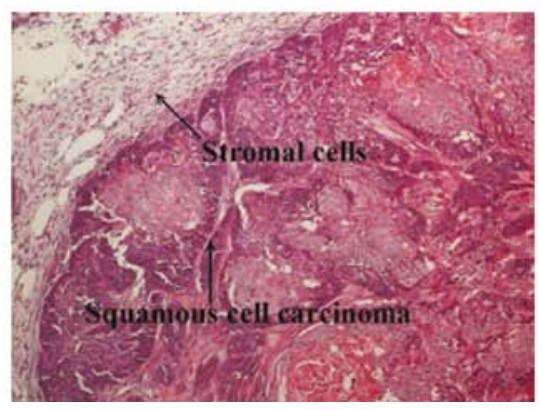

B
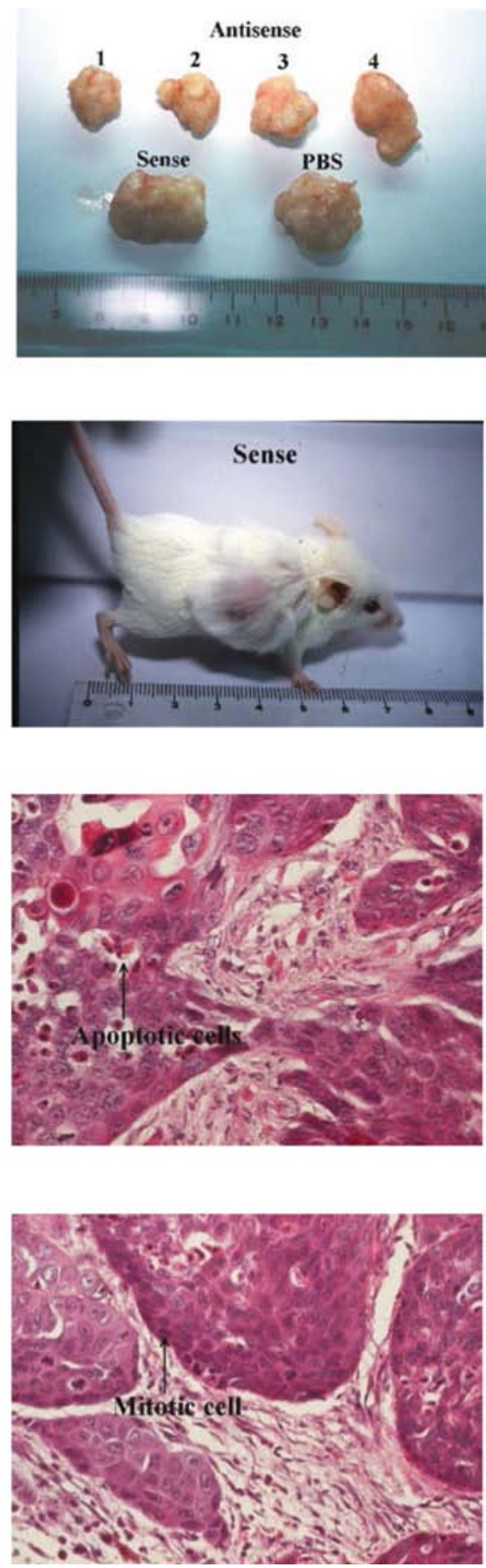

Figure 6. Nucleolin antisense oligodeoxynucleotides suppress NPC-TW01 xenograft tumor growth. Mice bearing NPC-TW01 xenograft tumors were randomly divided into three groups: antisense, sense and PBS. The tumor volume was measured and is shown in (A). After sacrificing the mice, the xenograft tumors were isolated (B). The mice bearing NPC xenograft tumors are shown (C). The xenograft tumors obtained from antisense oligodeoxynucleotide-treated mice are smaller than those of sense oligodeoxynucleotide-treated and PBS-treated mice. The NPC xenograft tumor sections from nucleolin antisense oligodeoxynucleotide- and nucleolin sense oligodeoxynucleotide-treated mice were analyzed using histopathological methods (H\&E stain). (D) In a mouse xenograft tumor treated with nucleolin antisense oligodeoxynucleotides, an ulcerated skin xenograft nodule display marked tumor necrosis (left panel: low magnification) with numerous apoptotic tumor cells (right panel: high magnification). (E) In a mouse xenograft tumor treated with nucleolin sense oligodeoxynucleotides, the xenograft tumor section show proliferating keratinizing squamous cell carcinoma (left panel: low magnification; right panel: high magnification).

and behavior of the mouse. Therefore, further investigation is required to obtain a detailed observation. In the current study, we only tested NPC-TW01 xenograft tumors. However, other cancer cell line-based xenograft tumors should be tested to evaluate the therapeutic effect of nucleolin antisense oligodeoxynucleotides in the future. 
In summary, our results show that nucleolin mRNA and protein expression levels are up-regulated in NPC cells, NPC biopsy and other cancer cell lines. Transfection of nucleolin antisense oligodeoxynucleotides induces apoptosis in NPC-TW01 cells and suppresses the growth of NPC-TW01 xenograft tumors. Nucleolin and nucleolin antisense oligodeoxynucleotides may have potential applications in nasopharyngeal carcinoma and other cancer therapies.

\section{Acknowledgements}

This study was supported in part by the National Science Council (NSC 100-2320-B-002-010, and NSC 100-2325-B-002-034) and by National Taiwan University Hospital (NTUH-99-A102) to C.-T.L.

\section{References}

1. Ferlay J, Shin HR, Bray F, Forman D, Mathers C and Parkin DM Estimates of worldwide burden of cancer in 2008: GLOBOCAN 2008. Int J Cancer 127: 2893-2917, 2010.

2. Lin CT, Chan WY, Chen W, et al: Characterization of seven newly established nasopharyngeal carcinoma cell lines. Lab Invest 68: 716-727, 1993.

3. Lin CT, Wong CI, Chan WY, et al: Establishment and characterization of two nasopharyngeal carcinoma cell lines. Lab Invest 62: 713-724, 1990

4. Lee YC, Hwang YC, Chen KC, et al: Effect of Epstein-Barr virus infection on global gene expression in nasopharyngeal carcinoma. Funct Integr Genomics 7: 79-93, 2007.

5. Mongelard F and Bouvet P: Nucleolin: a multiFACeTed protein. Trends Cell Biol 17: 80-86, 2007.

6. Bartova E, Horakova AH, Uhlirova R, et al: Structure and epigenetics of nucleoli in comparison with non-nucleolar compartments. J Histochem Cytochem 58: 391-403, 2010.

7. Srivastava M, Fleming PJ, Pollard HB and Burns AL: Cloning and sequencing of the human nucleolin cDNA. FEBS Lett 250 99-105, 1989.

8. Derenzini M, Sirri V, Trere D and Ochs RL: The quantity of nucleolar proteins nucleolin and protein B23 is related to cell doubling time in human cancer cells. Lab Invest 73: 497-502, 1995.

9. Roussel P and Hernandez-Verdun D: Identification of Ag-NOR proteins, markers of proliferation related to ribosomal gene activity. Exp Cell Res 214: 465-472, 1994.

10. Sirri V, Roussel P, Trere D, Derenzini M and HernandezVerdun D: Amount variability of total and individual Ag-NOR proteins in cells stimulated to roliferate. J Histochem Cytochem 43: 887-893, 1995

11. Bennett CF and Swayze EE: RNA targeting therapeutics: molecular mechanisms of antisense oligonucleotides as a therapeutic platform. Annu Rev Pharmacol Toxicol 50: 259-293, 2010.
12. Di Cresce C and Koropatnick J: Antisense treatment in human prostate cancer and melanoma. Curr Cancer Drug Targets 10: $555-565,2010$.

13. Wu HC, Huang PH, Chiu CY and Lin CT: G protein beta2 subunit antisense oligonucleotides inhibit cell proliferation and disorganize microtubule and mitotic spindle organization. J Cell Biochem 83: 136-146, 2001.

14. Hau P, Jachimczak P and Bogdahn U: Treatment of malignant gliomas with TGF-beta2 antisense oligonucleotides. Expert Rev Anticancer Ther 9: 1663-1674, 2009.

15. Tamm I: AEG-35156, an antisense oligonucleotide against $\mathrm{X}$-linked inhibitor of apoptosis for the potential treatment of cancer. Curr Opin Investig Drugs 9: 638-646, 2008.

16. Chi KN, Zoubeidi A and Gleave ME: Custirsen (OGX-011): a second-generation antisense inhibitor of clusterin for the treatment of cancer. Expert Opin Investig Drugs 17: 1955-1962, 2008.

17. Ito MK: ISIS 301012 gene therapy for hypercholesterolemia: sense, antisense, or nonsense? Ann Pharmacother 41: 1669-1678, 2007.

18. Liao SK, Perng YP, Shen YC, Chung PJ, Chang YS and Wang CH: Chromosomal abnormalities of a new nasopharyngeal carcinoma cell line (NPC-BM1) derived from a bone marrow metastatic lesion. Cancer Genet Cytogenet 103: 52-58, 1998.

19. Yao KT, Zhang HY, Zhu HC, et al: Establishment and characterization of two epithelial tumor cell lines (HNE-1 and HONE-1) latently infected with Epstein-Barr virus and derived from nasopharyngeal carcinomas. Int J Cancer 45: 83-89, 1990.

20. Cancer Institute, Laboratory of Tumor Viruses and Laboratory of Cell Biology, Chinese Academy of Medical Sciences and Chungshan Medical College: Establishment of an epithelial cell line and fusiform cell line from a patient with nasopharyngeal carcinoma. Sci Sin 21: 113-132, 1978.

21. Chu YW, Yang PC, Yang SC, et al: Selection of invasive and metastatic subpopulations from a human lung adenocarcinoma cell line. Am J Respir Cell Mol Biol 17: 353-360, 1997.

22. Chen CM, Chiang SY and Yeh NH: Increased stability of nucleolin in proliferating cells by inhibition of its self-cleaving activity. J Biol Chem 266: 7754-7758, 1991.

23. Sengupta TK, Bandyopadhyay S, Fernandes DJ and Spicer EK: Identification of nucleolin as an AU-rich element binding protein involved in bcl-2 mRNA stabilization. J Biol Chem 279: 10855-10863, 2004.

24. Otake Y, Sengupta TK, Bandyopadhyay S, Spicer EK and Fernandes DJ: Drug-induced destabilization of bcl-2 mRNA: a new approach for inducing apoptosis in tumor cells. Curr Opin Investig Drugs 5: 616-622, 2004.

25. Otake Y, Soundararajan S, Sengupta TK, et al: Overexpression of nucleolin in chronic lymphocytic leukemia cells induces stabilization of bcl2 mRNA. Blood 109: 3069-3075, 2007.

26. Takagi M, Absalon MJ, McLure KG and Kastan MB: Regulation of p53 translation and induction after DNA damage by ribosomal protein L26 and nucleolin. Cell 123: 49-63, 2005.

27. Ugrinova I, Monier K, Ivaldi C, et al: Inactivation of nucleolin leads to nucleolar disruption, cell cycle arrest and defects in centrosome duplication. BMC Mol Biol 8: 66, 2007. 